\title{
LECTURE
}

\section{Neuromonitoring in Operations in the Skull Base}

\author{
Aage R Møller \\ Department of Neurological Surgery, University of Pittsburgh School of Medicine, Pittsburgh, PA USA
}

(Received for publication on April 30, 1991)

\begin{abstract}
Methods to monitor the integrity of cranial motor nerves during operations on skull base tumors have been developed over the past decade. These methods can help the surgeon to identify cranial motor nerves that are located in the surgical field but which may not be visible directly. Methods have also been developed that allow monitoring of the function of sensory systems such as the auditory system and it has been shown that brainstem auditory evoked potentials can provide important information about the integrity of the auditory nervous system. It has been shown in several studies that such neuromonitoring, when performed during operations in the skull base, can help reduce the incidence of permanent neurological deficits that may occur with the removal of large tumors. We have also shown that such monitoring can be performed routinely without interfering noticeably with the actual surgical procedures. (Keio J Med 40 (3): 151-159, September 1991)
\end{abstract}

Key words: neurophysiological monitoring, intraoperative monitoring, skull base tumors

\section{Introduction}

Intraoperative neuromonitoring of evoked potentials is a relatively new addition to the armamentarium of techniques available to reduce the incidence and/or severity of complications of operations in which specific parts of the nervous system are manipulated. During the past 15 to 20 years techniques that are based on neurophysiological recordings have begun to be used during neurosurgical, otolaryngological, and orthopedic operations. ${ }^{1-14}$ It is generally agreed that these new techniques are valuable and that they can help reduce complications in the form of neurological deficits. Intraoperative electrophysiological recordings have also proven to be able to aid the surgeon in the operation in that 1 ) the information provided by such recordings can help identify neural structures, ${ }^{6-8,14}$ and 2) neurophysiological recordings may in a few cases provide information that is of value in reaching the therapeutic goal of the operation and in evaluating the success of the operation before the operation is completed. ${ }^{15}$

It is difficult to obtain formal proof that the use of intraoperative neuromonitoring reduces the complications and improves the outcome of specific operations, but it is generally agreed by those who have used these methods that these new methods are indeed very helpful. It is the opinion of surgeons who have used these methods for some time that neuromonitoring can decrease the risks of surgically induced injuries that may result in permanent neurological deficits, as well as help the surgeon in various ways to carry out the operation. These benefits in fact are among the reasons why it has been difficult to provide proof of the efficacy of intraoperative neuromonitoring in reducing complications in the form of neurological deficits or in improving the outcome of the operations: It has generally not been possible to arrange studies in which patients are randomly assigned to undergo monitoring because of humanitarian reasons and because the surgeons in charge will not allow resources such as neuromonitoring to be provided to their patients by random selection, as is necessary for such studies. A few do detail the incidence of neurological deficits after neuromonitoring was introduced (see, e.g. ${ }^{10,16}$ ) and by comparing such results with the rate of deficits before monitoring it is possible to obtain an estimate of the benefits from monitoring. The actual improvement in the incidence of neurological deficits attributable to neuromonitoring cannot be determined exactly, however, because other technological advances that have been introduced in the same time period have

Reprint requests to: Dr Aage R Møller, Department of Neurological Surgery, 9402 Presbyterian-University Hospital, 230 Lothrop Street, Pittsburgh, PA 15213, USA 
undoubtedly contributed to the reduced incidence of neurological deficits.

In the present paper we describe methods by which it is possible to monitor the function of cranial motor nerves intraoperatively, and we also show how intraoperative monitoring of one sensory system in particular, namely the auditory system, can be useful in operations in the skull base.

\section{Intraoperative Monitoring of Cranial Motor Nerves}

In operations in the skull base, particularly those that involve removal of tumors from the cavernous sinus, several cranial motor nerves are at risk. ${ }^{11}$ Intraoperative monitoring of the electromyographic (EMG) potentials that can be recorded from the muscles that these nerves innervate can help to identify these nerves when the anatomy is altered by a tumor or when a tumor has so involved one or more of these nerves that the nerves cannot be identified on the basis of knowledge of normal anatomy. In such cases, it is necessary to be able to stimulate these motor nerves electrically in the surgical field while recording EMG potentials from the muscles that these nerves innervate. The motor nerves can be stimulated electrically by applying rectangular electrical impulses delivered by a handheld monopolar electrode.

To obtain optimal benefit from recording the responses from the muscles that these nerves innervate, electrodes should be placed in or close to the respective muscles that are innervated by these nerves and the recorded potentials must be displayed in appropriate forms in the operating room. ${ }^{7,8.17}$ Evaluating potentials recorded in this way can also help to reduce the risks of injuring these nerves. For such evaluation it is of special importance to monitor EMG activity that is elicited by surgical manipulations that affect the nerve in question.

In order to be able to record EMG potentials from any muscle, it is necessary that the patient not be paralyzed during the operation. This is because it is impossible to record EMG potentials from a patient who is paralyzed. Because the most often used anesthesia regimen is the so-called balanced anesthesia regimen in which the patient is given a strong narcotic, nitrous oxide, and a muscle-relaxant type endplate blocker, maintaining anesthesia in the patient undergoing neuromonitoring, which can only be performed without using musclerelaxing agents, usually involves a change from the normal anesthetic procedure. To be able to record muscle responses 1) an inhalation anesthesia regimen that uses agents such as isoflurane or halothane must be used or 2) anesthetic agents that can be administed intravenously to obtain surgical anesthesia, such as barbiturates or, e.g. agents like propofol must be used. Common to most of these alternatives to balanced anesthesia is that they are more complex to use than balanced anesthesia.

\section{Monitoring the facial nerve in operations for acoustic tumors}

Loss of facial function is a devastating complication of removal of acoustic tumors. The risk of this complication has been reduced considerably, however, through the introduction of microsurgical techniques in the 1970s and more recently through the introduction of intraoperative monitoring of facial nerve function in connection with electrical stimulation of the tumor. Different methods have been used to identify contractions of the facial muscles. The first method used was to have an assistant hold his/her hand on the face of the patient and to feel for muscle contractions. Visual observation has also been used to identify contractions of facial muscles in such patients, but it was an important step forward when methods were developed that allow for automatic recording and display of facial muscle contractions. One of the first such methods to be described was that of Sugita and Kobayashi, ${ }^{14}$ who used accelerometers placed on the face to signal a contraction of the facial musculature. Later Delgado et al ${ }^{18}$ introduced the use of electromyography to record potentials from face muscles. This method was further developed by Møller and Jannetta ${ }^{8}$ and M $\varnothing l l e r,{ }^{7}$ who also demonstrated the advan tages of making the recorded EMG signal audible so that the operating surgeon can monitor facial muscle contractions just by listening to the sound of the facial muscle contractions. We also suggested that it might be of value to use this technique not only to identify the facial nerve but, more importantly, also to identify areas of the tumor where there is no facial nerve present so that these portions can be removed safely without the risk of injuring the facial nerve. Using EMG potentials in this way has also reduced the duration of such operations considerably.

In order to be able to use facial EMG potential recording not only for identifying the facial nerve but also for identifying areas of the tumor where there is no facial nerve present, the electrical stimulation must be so arranged that it always will activate the facial nerve and thereby result in an EMG response if the stimulating electrode is sufficiently close to the nerve. It is therefore important that the electrical current used to stimulate the facial nerve be as little influenced as possible by changes in the electrical conductivity of the medium that surrounds the nerve. Traditionally, electrical stimulation of nervous tissue is via delivery of a constant current rather than a constant voltage because keeping the current constant reduces the effect of varying electrode impedance. When stimulating a tumor electrically in order to identify the facial nerve (or to identify areas of the tumor where there is no nerve), however, variation in electrode impedance is not the major problem. Rather, variations in the degree to which electrical current is 
shunted through varying amounts of cerebrospinal fluid (CSF) covering the electrode is the dominant variable in that situation. The effect of such variations in shunting can be reduced by using stimulation of the constantvoltage type. Ideally, the strength of a constant-voltage stimulus to a nerve that is hidden in tissue, such as that of a tumor, is independent of the degree of electrical shunting; however, for practical purposes a semiconstant voltage is most practical. ${ }^{7,8}$

Another consideration in facial EMG potential recording is the type of stimulating electrode used. It is customary to use bipolar electrodes for stimulation of nerves of the central nervous system. However, a monopolar electrode (Figure 1) is more suitable for the purpose of identifying a facial nerve that has been engulfed by a tumor, and in particular a monopolar stimulating electrode is most suitable for the purpose of

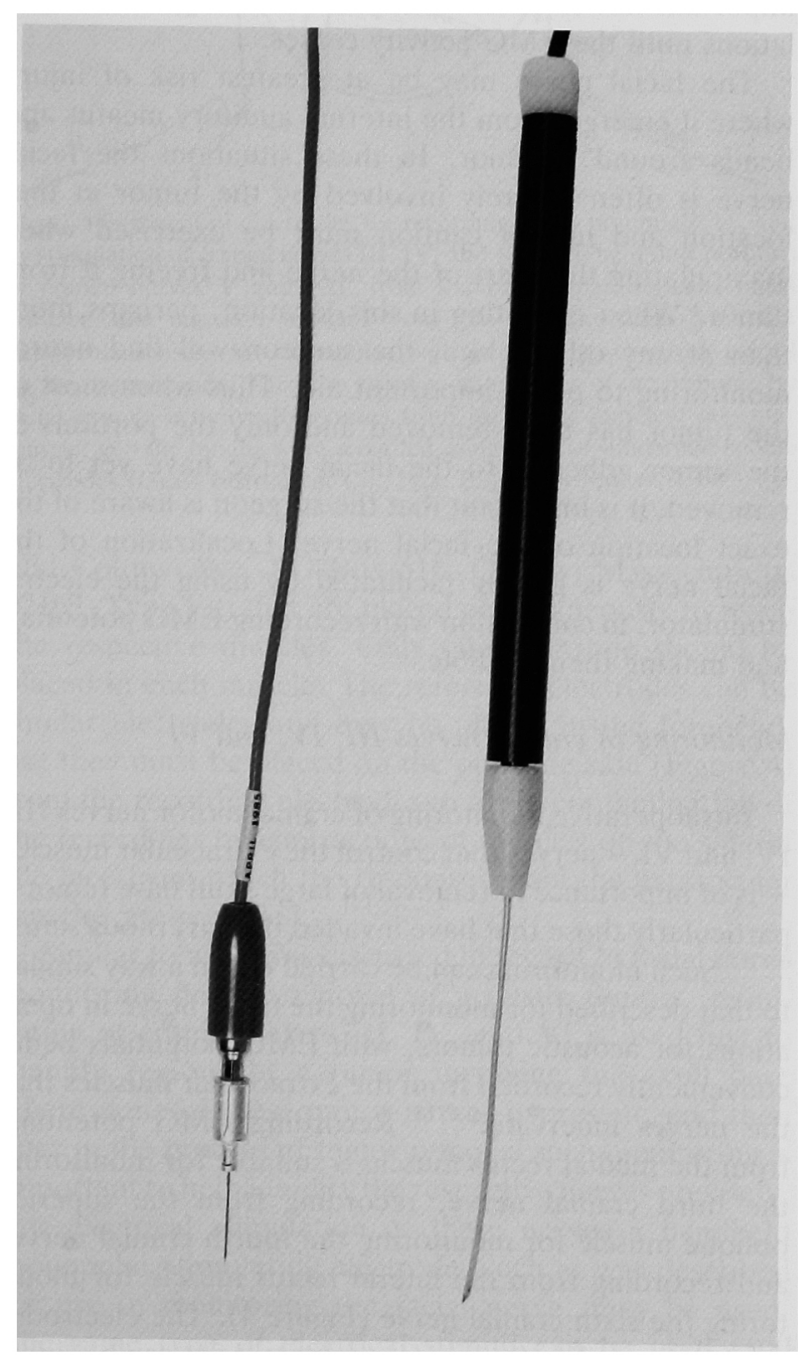

Fig 1 Handheld monopolar stimulating electrode and hypodermic needle used as return electrode (from Møller, 19887). identifying regions of a tumor where there is no portion of the facial nerve present. ${ }^{7,8}$ This is true because the efficiency with which a monopolar electrode stimulates a nerve that is located within a tumor is not dependent on the orientation of the electrode, as it is when a bipolar electrode is used. Also, and not least important, a monopolar electrode is easy to use, and when it is supplied with a semiconstant-voltage stimulus source it can deliver a stimulus that is relatively little affected by how much CSF is present in the operative field.

Rectangular impulses of a duration of about $100 \mu \mathrm{S}$ are suitable for probing an acoustic tumor for the presence of a portion of the facial nerve as well as for stimulation of the facial nerve in general.

When we wish to detect contraction of any of the facial muscles that are innervated by the facial nerve, we place EMG recording electrodes with one on the upper face and one on the lower face (Figure 2). By recording differentially from these two electrodes contraction of any one of the facial muscles will give rise to a recorded potential (Figure 3). This naturally also results in recording of the activity of the masseter and temporal muscles that are innervated by the motor portion of the fifth cranial nerve. Because the latency of that response

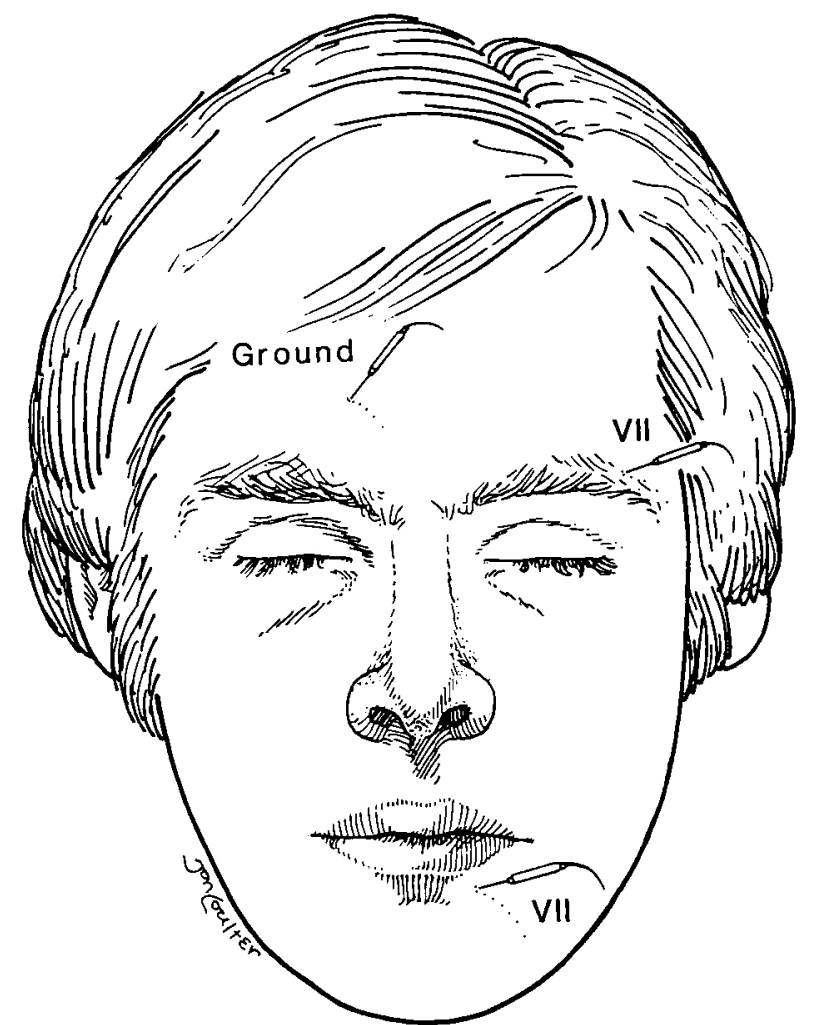

Fig 2 Schematic illustration of the placement of electrodes for recording EMG potentials from face muscles. The electrodes are platinum needles (Grass Instrument Co, Type E2) and are placed subdermally
(from Møller, 1988 ${ }^{7}$ ). 


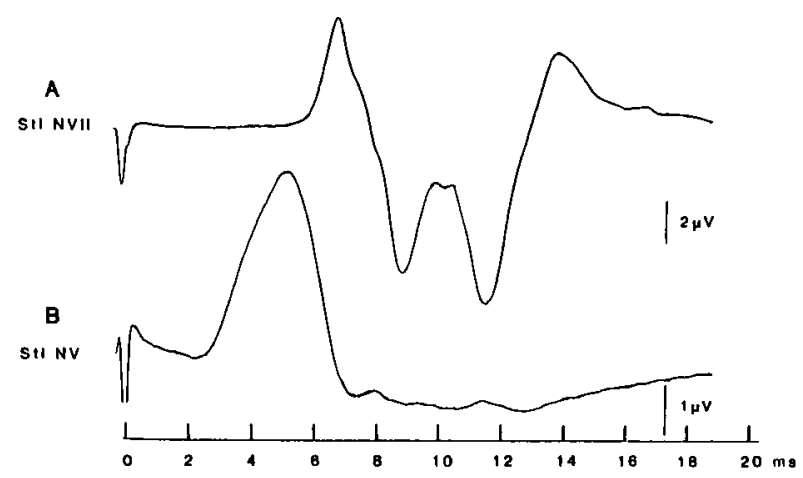

Fig 3 Typical recordings from electrodes placed in the face as illustrated in Figure 2. A: Obtained when the intracranial portion of the facial nerve was stimulated electrically with rectangular impulses of $100-\mu \mathrm{S}$ duration and a voltage of about $0.8 \mathrm{~V}$. B: Obtained when the motor portion of the fifth cranial nerve was stimulated electrically with similar pulses (from Møller, 19887).

is only about $1.5 \mathrm{~ms}^{7}$ and the latency of the response from the facial nerve is about $5 \mathrm{~ms},{ }^{19}$ by viewing the response on an oscilloscope it is possible to differentiate between the response elicited by stimulation of the fifth nerve and the response to stimulation of the facial nerve (Figure 3).

Making the EMG potentials audible $e^{7,8,17}$ is of great value for the purpose of making communication between the neurophysiologist and the surgeon as easy as possible. It also makes it possible for the surgeon him/herself to determine if there is a response or not when he/she is probing the tumor with the stimulating electrode.

The purpose of probing the tumor with the electrical stimulator in the beginning of the operation is to identify areas of the tumor that are free of facial nerve. These parts of the tumor can then be removed without risk of injuring the facial nerve. This use of the stimulator makes the first part of the tumor removal process more rapid than would have been the case without intraoperative neuromonitoring. When enough tumor has been removed in this way that the facial nerve can be identified, either visually or by electrical stimulation, it is important that the surgeon at all times keep track of exactly where the facial nerve is located so that injury to it can be avoided. When the facial nerve cannot be identified visually and therefore must be identified by electrical stimulation, it is again very important that the stimulating electrode is easy to use so that it can be used frequently to verify the location of the facial nerve. In the case of a large tumor in which the facial nerve cannot be identified visually and where it has more the appearance of wet tissue paper than of a nerve, it is even more important to use electrical stimulation frequently so that the location of the facial nerve is known to the surgeon at all times. Electrical stimulation seems to be the only practical way to determine the location of the facial nerve in such situations.

Once the facial nerve has been identified with the help of electrical stimulation, it becomes important to protect the facial nerve from injuries that can occur with its manipulation in connection with tumor removal. An important way to reduce the risk of permanent injury to the facial nerve is monitoring the EMG activity that may result from such manipulation. Such EMG activity may occur only while the nerve is being manipulated or it may persist for seconds or minutes after the manipulation of the nerve has stopped, with the latter condition being more likely than the former to indicate that the manipulation will result in transient or permanent postoperative facial weakness. In any case, if surgical manipulation of the facial nerve results in such EMG activity it should be taken as an indication that the surgeon needs to be more gentle or to change the operative technique. When EMG activity persists after the manipulation has stopped, it is important not to perform any further surgical manipulations until the EMG activity ceases.

The facial nerve may be at greatest risk of injury where it emerges from the internal auditory meatus and bends around a tumor. In these situations the facial nerve is often severely involved by the tumor at that location and utmost caution must be exercised when manipulating that part of the nerve and freeing it from tumor. When operating in this location, perhaps more than at any other place, the surgeon will find neuromonitoring to be an important aid. Thus when most of the tumor has been removed and only the portions of the tumor adhering to the facial nerve have yet to be removed, it is important that the surgeon is aware of the exact location of the facial nerve. Localization of the facial nerve is greatly facilitated by using the electric stimulator, in connection with recording EMG potentials and making them audible.

\section{Monitoring of cranial nerves $I I I, I V$, and $V I$}

Intraoperative monitoring of cranial motor nerves III, IV, and VI - nerves that control the extraocular muscles - is of importance in removal of large skull base tumors, particularly those that have invaded the cavernous sinus. $6,7,11$ Such monitoring can be carried out in a way similar to that described for monitoring the facial nerve in operations for acoustic tumors, with EMG potentials being conveniently recorded from the extraocular muscles that the nerves innervate. ${ }^{6,7,11}$ Recording EMG potentials from the medial rectus muscle is suitable for monitoring the third cranial nerve, recording from the superior oblique muscle for monitoring the fourth cranial nerve, and recording from the lateral rectus muscle for monitoring the sixth cranial nerve (Figure 4 ). The electrodes used for recording from extraocular muscles can be single subdermal needle electrodes (Grass Instrument Co, 101 


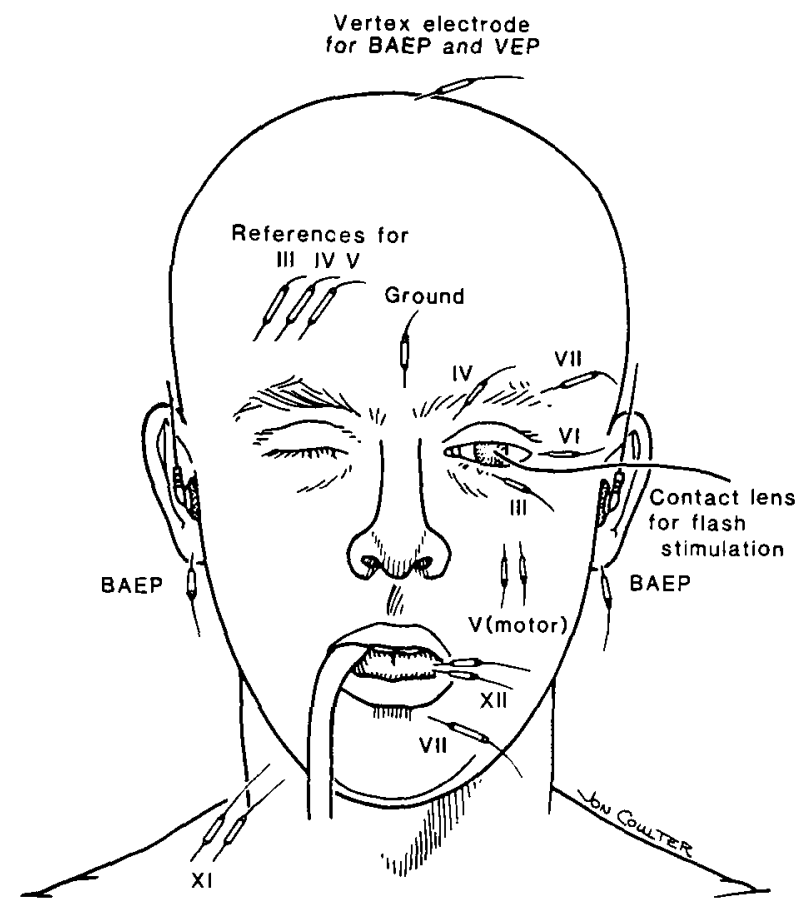

Fig 4 Placement of elcctrodes for recording EMG potentials, elicited by stimulation of cranial nerves III, IV, and VII and the motor portions of cranial nerves $\mathrm{V}$ and XII, from the extraocular muscles, facial muscles, and masseter muscle. Note that the recordings from the extraocular muscles are monopolar, with the reference electrode placed on the opposite side. This is to avoid contamination of the response by facial muscle activity. Responses from the facial muscles, masseter muscle, and the tongue were recorded using pairs of subdermal needle electrodes (Grass Instrument Co, Type E2) (from Møller, 19887).

Old Colony Ave, PO Box 516, Quincy, Massachusetts 02169, Type E2) that are placed percutaneously to reach the respective muscles. Only one electrode should be placed in each muscle. The reference electrodes can be similar electrodes and may be placed in the forehead, but they must be placed on the opposite side (Figure 4) from the recording electrodes to avoid contamination of the recordings by contractions of muscles on the side of the face from which the responses from the extraocular muscles are recorded.

Similar to what was discussed in regard to facial nerve monitoring during removal of acoustic tumors, monitoring of cranial nerves III, IV, and VI is used first to identify regions of a tumor involving the skull base where none of these motor nerves is present, and then later in the process of tumor removal such monitoring is important to help identify the respective nerves precisely. For electrical stimulation of these nerves a handheld monopolar stimulating electrode, such as was described for use in monitoring the facial nerve, may be used. The same type of electrical stimuli can be used $(100$ to $150-\mu \mathrm{S}$ duration rectangular pulses, semiconstant voltage), but to stimulate the fourth cranial nerve in particular, and sometimes the third and sixth cranial nerves, a slightly higher stimulus strength $(1.5$ to $2 \mathrm{~V}$ versus 0.5 to $1.0 \mathrm{~V}$ ) may be needed than is adequate to stimulate the facial nerve. Typical examples of recordings from the extraocular muscles as well as from the facial muscles are shown in Figure 5. Just as monitoring spontaneous activity of the facial nerve can help prevent its injury in operations for acoustic tumors, monitoring of spontaneous activity of the nerves that innervate the extraocular muscles can help to prevent injury to cranial nerves III, IV, and VI because the presence of spontaneous activity may indicate that injury is being caused by manipulation of any of these nerves.

\section{Monitoring of other cranial motor nerves}

In operations that involve the caudal brainstem it can be of value to monitor the twelfth cranial nerve and also in some cases the eleventh cranial nerve. Such monitoring can be performed easily by recording EMG potentials from the respective muscles that these nerves innervate. For monitoring the twelfth nerve electrodes are placed in the tongue, and for monitoring the eleventh nerve electrodes are placed in either one of the muscles that are innervated by that nerve (e.g. the trapezoidal muscle). ${ }^{7}$ Bipolar recordings can easily be obtained by placing pairs of electrodes in the muscles (e.g. Grass Instrument $\mathrm{Co}$, Type E2, subdermal needle electrodes similar to those used for recording from the facial muscles) (Figure 4). The potentials that can be recorded in this way are of large amplitude so that they can easily be displayed on an oscilloscope without any averaging, and they can be made audible so that each member of the operating room team can hear the potentials and any changes that occur.

\section{Monitoring of brainstem auditory evoked potentials (BAEP)}

Intraoperative monitoring of BAEP has been shown to be of benefit in many types of operations. ${ }^{1-3,9,16.20-23}$ When the purpose of monitoring BAEP is to preserve hearing, the benefits of that technique are particularly obvious. This is true especially for operations in the cerebellopontine angle (CPA), such as microvascular decompression (MVD) operations to relieve trigeminal neuralgia (TN), hemifacial spasm (HFS), or disabling positional vertigo (DPV). In such operations, during which the eighth cranial nerve is exposed, it is also beneficial to record the compound action potentials (CAP) directly from the eighth nerve. ${ }^{7,20.23}$

In the operating room it is practical to use small earphones that can fit in the ear canal to deliver the 

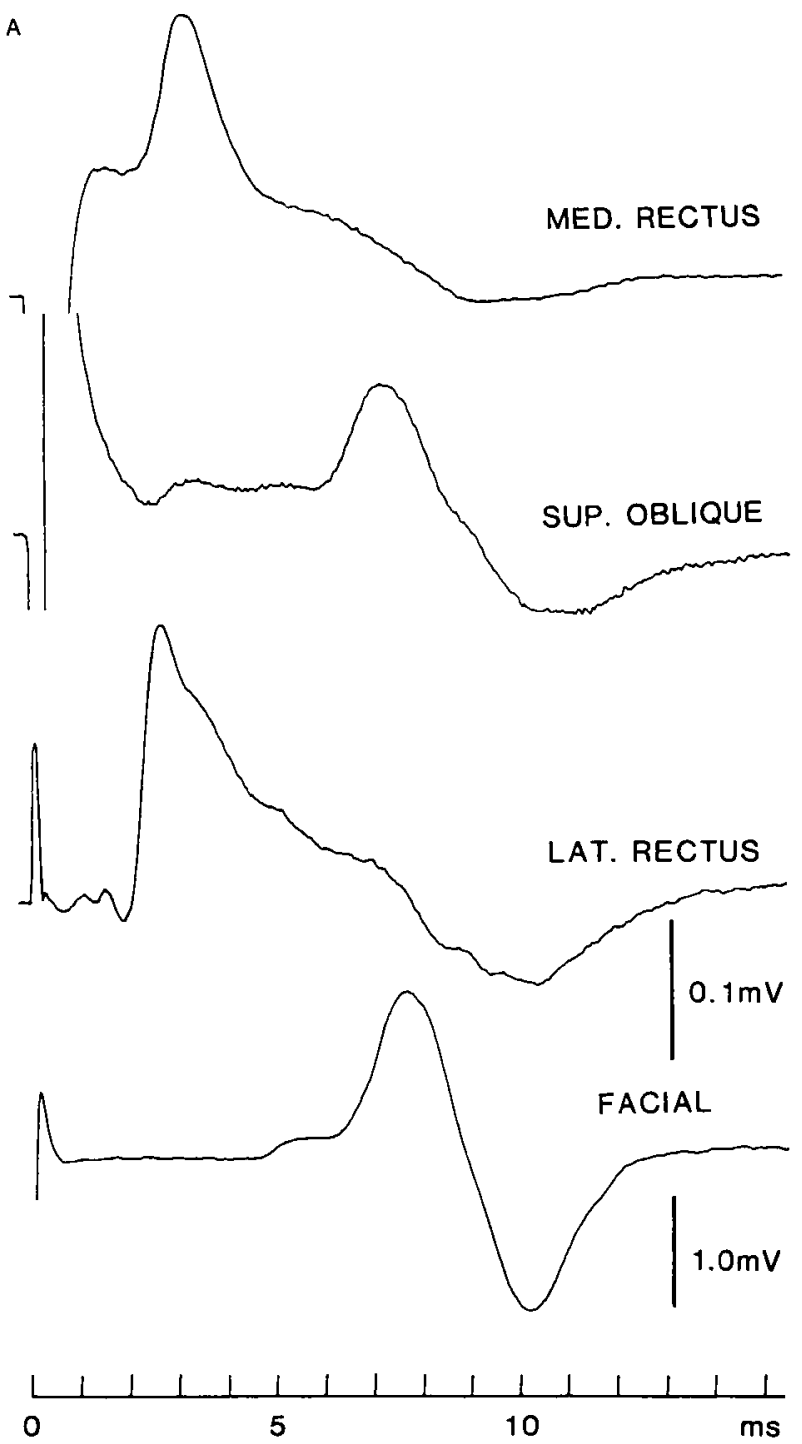

B

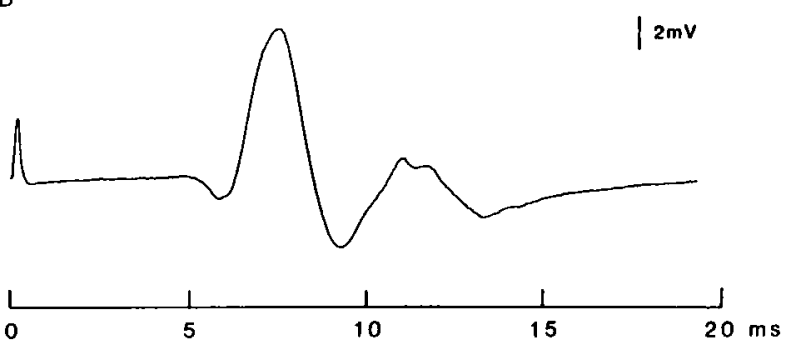

Fig 5 A: Typical EMG responses recorded from the extraocular muscles and the facial muscles using the electrode placement illustrated in Figure 4. B: Recordings similar to those in A, but obtained from the tongue via a pair of needle electrodes (from Møller, 19887). sound stimuli for evoking the auditory responses. We use miniature stereo earphones that are designed to be used together with portable radios or tape recorders. ${ }^{7}$ These earphones can be sealed watertight in the ear canal with adhesive plastic tape (3 M Co. Minnesco Division/ 3 M, 224-4 NW, 3 M Center, St Paul, Minnesota 55144, Blenderm) so that the earphones are not dislodged during the operation and so that fluid cannot reach the ear canal or the earphone during the operation. These earphones are mechanically stable and are not broken by the rough treatment equipment sometimes is subject to in the operating room. It is practical to use click stimuli for intraoperative monitoring of auditory evoked potentials, and these earphones can deliver such click sounds when rectangular electrical impulses are applied to them. Such pulses can be delivered by most auditory stimulators that are designed to be used in connection with BAEP monitoring.

When BAEP are recorded from electrodes placed on the scalp in the traditional way, namely differentially between an electrode placed at the vertex and one on the earlobe, the recorded auditory evoked potentials are of very low amplitude, much lower than the amplitude of background electrical activity (which is dominated by EEG activity but also includes electrical interference signals). Such background activity is particularly prominent in the operating room where there typically are numerous sources of electrical interference. Two methods can be used to recover auditory evoked potentials from this background of signals, 1) signal averaging, in which many responses to the same stimulus are added, or 2) spectral filtering of the responses.

With signal averaging, responses that appear with the same temporal relationship to the stimulus will add. Signals, such as EEG signals, that appear randomly, however, will not increase in amplitude with time. The actual ratio between the amplitude of the signal that has the same temporal relation to the stimulus (the evoked response) and the amplitude of background noise will increase with the square root of the number of responses that are added.

With the second method of improving the signal-tonoise ratio, spectral filtering of the recorded potentials, two factors should be taken into consideration. The usefulness of spectral filtering is based on the assumption that the spectral distribution of energy of the evoked potentials and the background noise was different, and therefore parts of the energy spectrum that were outside the spectrum of the evoked potentials could be removed without interfering with the evoked potentials. The first factor that complicates the use of spectral filtering, however, is that when traditional electronic filters are used distortion of the waveform of the evoked potentials will occur even if the energy of the evoked potentials is only attenuated negligibly, and the location of the various 
peaks of an evoked potential may be shifted in time as a result of the filtering. This is because of the unavoidable phase shift that is associated with electronic filters. This phase shift occurs because electronic filters must always work on the past history of the signal that is to be filtered. Modern computer technology, however, allows a different type of filtering, one that does not need to be physically realizable as do electronic filters. These "digital filters" can be designed to have no phase shift at all. $^{7,24}$ Further, digital filters can be designed without other constraints that electronic filters have, so that digital filters can therefore perform filtering that is by far more suitable for evaluating potentials than filtering performed by electronic filters.

The second factor that should be taken into consideration with spectral filtering of recorded potentials is that one does not always need to evaluate the entire energy spectrum of evoked potentials, such as BAEP, to interpret these potentials. When BAEP are recorded in the traditional way, namely differentially between the vertex and the earlobe or mastoid, the recording contains lowfrequency components that are not at all necessary for the interpretation of these potentials. ${ }^{24}$ Because it is the latencies of the different (vertex-positive) peaks that are regarded as the most important feature of these potentials, these unnecessary spectral components can be attenuated. Attenuation of low-frequency components increases the signal-to-noise ratio further than would have been the case if traditional filtering was applied, under the assumption that all the energy of the evoked potentials must be preserved. It is also possible to design filters that enhance the peaks in addition to attenuating the background noise. Figure 6 shows examples of BAEP before and after filtering with such zero-phase digital filters. The filtering enhances the peaks in the BAEP without causing any shift in the location of the peaks, and produces a "clean" waveform which lends itself to automated identification of the peaks (Figure 6A). It may be seen from Figure $6 B$ that, in a situation where the raw averaged responses do not allow identification of peaks in the BAEP other than peak V, the record that has been filtered by a digital filter (W50 in Figure 6B) is clearly interpretable and readily replicable. ${ }^{7}$

Although such digital filtering decreases the time it takes to obtain an interpretable BAEP waveform, there are advantages in being able to record the CAP from the exposed intracranial portion of the eighth nerve using an electrode made from a Teflon-insulated, multistrand, silver wire (Medwire Corp, 121 South Columbus Ave, Mt Vernon, New York 10553, Type 7/40T) with a small cotton wick sutured to its uninsulated tip. ${ }^{7.23} \mathrm{CAP}$ recorded from an electrode placed on the exposed eighth nerve (Figure 7) can usually be viewed directly on an oscilloscope without any averaging because the amplitudes of such responses are much larger than the ampli-

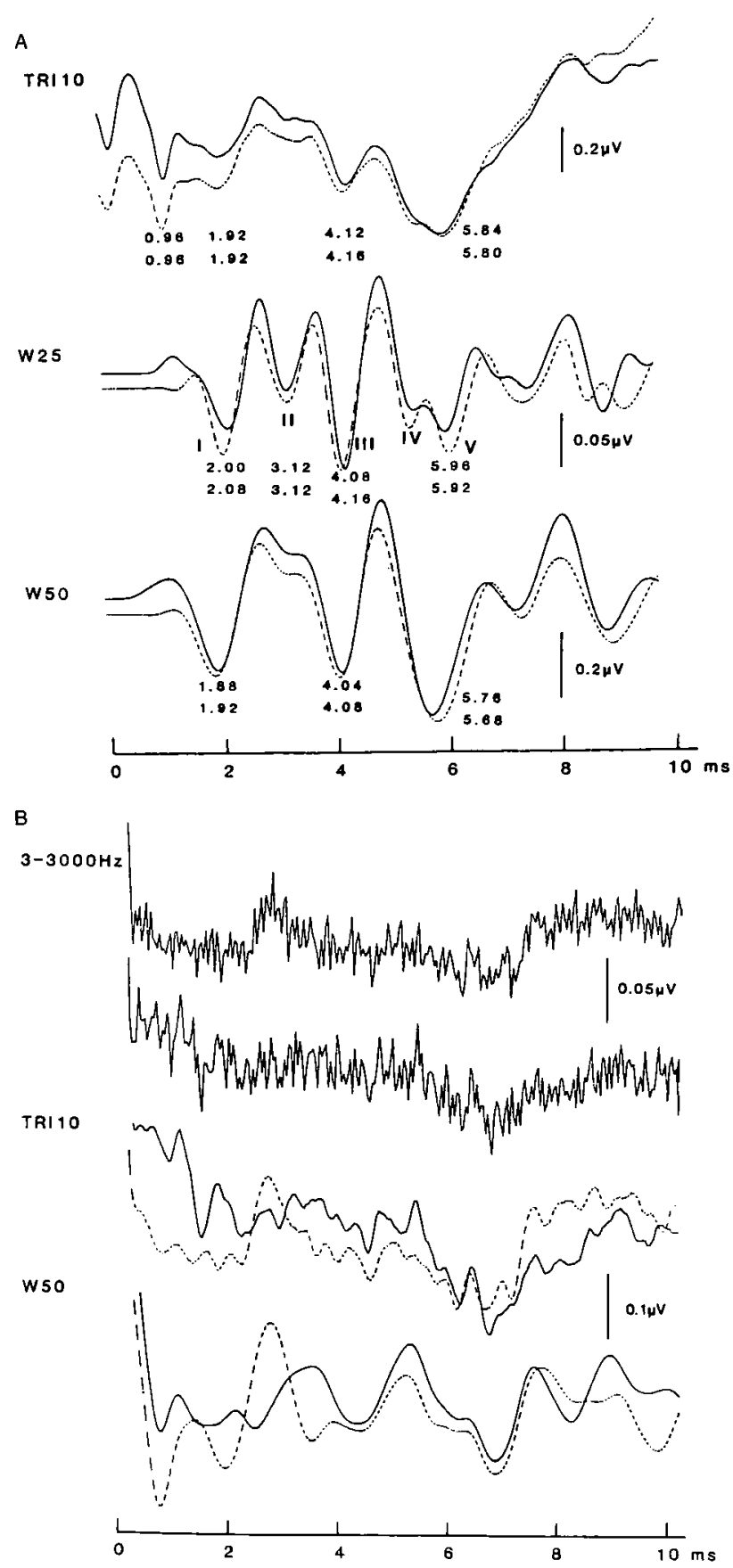

Fig 6 A: Effects of filtering with different types of zero-phase digital filters on BAEP waveforms recorded in patients with normal hearing. $B$ : Effects of filtering, with the same zero-phase digital filters used to obtain results in $\mathrm{A}$, noisy recordings of BAEP made under unfavorable conditions in the operating room (from Møller, $1988^{24}$ ).

tudes of the background noise (Figure 8). When injury to the auditory nerve causes a change in the recorded potentials, the directly recorded CAP provide more information than BAEP about the nature of the injury. 
A

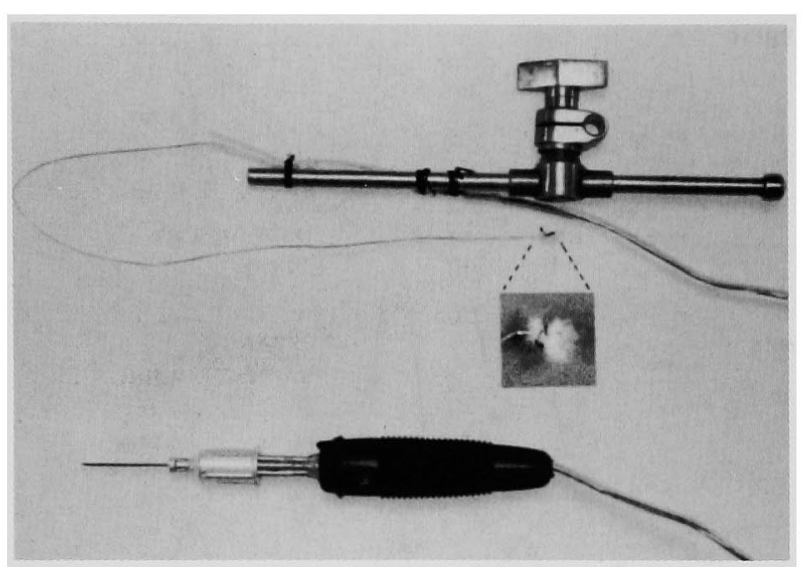

B

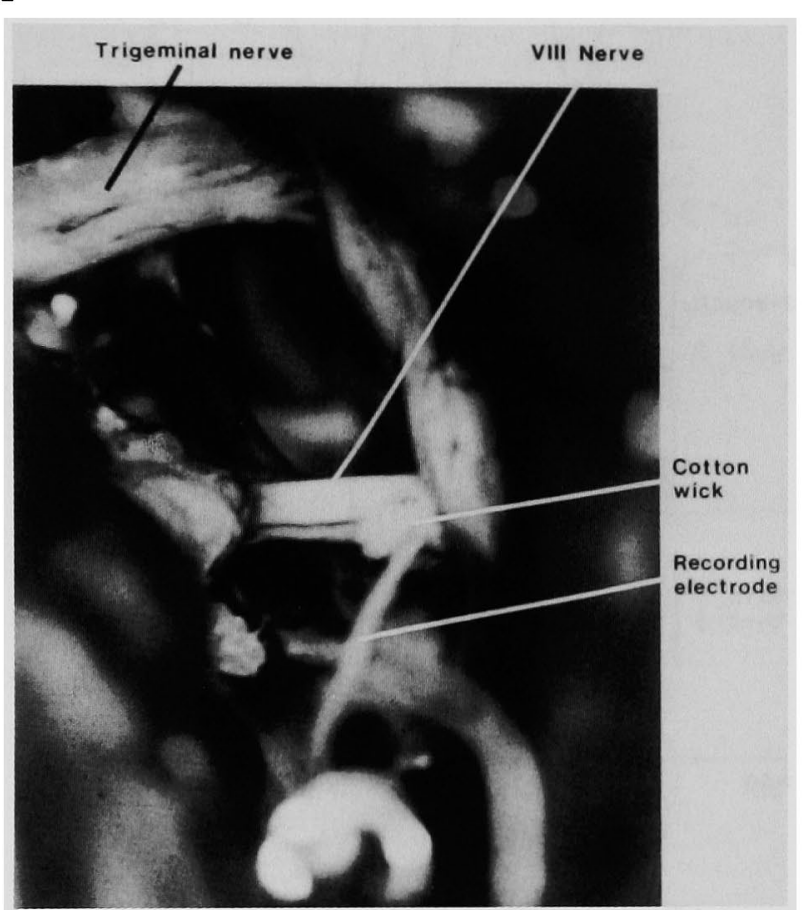

Fig 7 A: Electrode used to record CAP from the eighth nerve (from Møller, 1988 ${ }^{7}$ ). B: Placement of the recording electrode (shown in A) on the exposed intracranial portion of the eighth nerve (from Møller, $1988^{7}$ ).

Thus it may be seen from the lower tracing in Figure 8 that the waveform changes to a nearly single positive deflection, which is an indication of a nearly total conduction block, with heating from bipolar coagulation. When using such directly recorded CAP to detect injury to the eighth nerve, it must be kept in mind that only injuries to the portion of the nerve that is distal to the site of recording can be detected using that method.

Naturally it is only possible to record such CAP directly

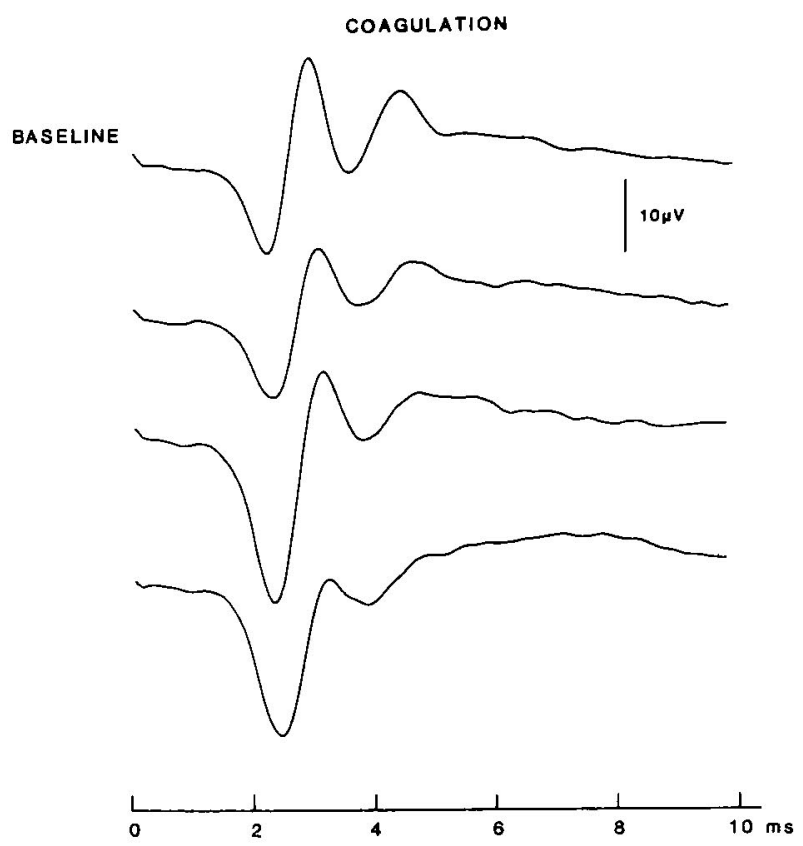

Fig 8 Top tracings show CAP recorded from the exposed eighth nerve, with negativity shown as an upward deflection. The triphasic waveform is typical for recordings from a long nerve with a monopolar electrode. The lower tracing shows the effect of heating due to bipolar coagulation (from Møller, 19887).

from the eighth nerve in operations where the eighth nerve is being exposed. In such operations, however, it is often advantageous to obtain an interpretable waveform in a short time, because in such operations the eighth nerve is usually being manipulated directly and therefore is at higher risk of injury than in operations in which it is only indirectly manipulated by such activities as retraction of brain tissue.

\section{Summary}

The author's experiences over the past 6 to 8 years in using the type of neuromonitoring described in this paper during operations in the skull base have shown that this type of monitoring not only decreases the risks for permanent neurological deficits but also makes the work of the surgeon easier and gives the surgeon a sense of security because neural structures that are not visible with the operating microscope can be identified using this method and injuries can be detected instantaneously.

\section{References}

1. Grundy BL: Monitoring of sensory evoked potentials during neurosurgical operations: Methods and applications. Neurosurgery 11: $556-572,1982$

2. Grundy BL: Intraoperative monitoring of sensory evoked poten- 
tials. Anesthesiology 58: 72-87, 1983

3. Grundy BL: Electrophysiologic monitoring: EEG and evoked potentials. In: Manual of Neuroanesthesia, Newfield P, Cottrell J, eds, Little Brown, Boston, 1983, 28-59

4. Grundy BL: Evoked potentials monitoring. In: Monitoring in Anesthesia and Critical Care Medicine, Blitt CD, ed, Churchill Livingstone, New York, 1985, 345-411

5. Friedman WA, Kaplan BJ, Gravenstein D, Rhoton AL: Intraoperative brain-stem auditory evoked potentials during posterior fossa microvascular decompression. J Neurosurg 62: 552-557, 1985

6. Møller AR: Electrophysiological monitoring of cranial nerves in operations in the skull base. In: Tumors of the Cranial Base: Diagnosis and Treatment, Sckhar LN, Schramm VL, cds, Futura Pub Co, Mt Kisco, New York, 1987, 123-132

7. Møller AR: Evoked Potentials in Intraoperativc Monitoring, Williams \& Wilkins, Baltimore, 1988

8. Møller AR, Jannetta PJ: Preservation of facial function during removal of acoustic neuromas: Usc of monopolar constant-voltage stimulation and EMG. J Neurosurg 61: 757-760, 1984

9. Raudzens PA: Intraoperative monitoring of evoked potentials. Ann NY Acad Sci 388: 308-326, 1982

10. Schramm J, Mokrusch T, Fahlbusch R, Hochstetter A: Detailed analysis of intraoperative changes monitoring brain stem acoustic evoked potentials. Neurosurgery 22: 694-702, 1988

11. Sekhar LN, Møller AR: Operative management of tumors involving the cavernous sinus. J Neurosurg 64: 879-889, 1986

12. Daspit CP, Raudzens PA, Shetter AG: Monitoring of intraoperative auditory brain stem responses. Otolaryngol Head Neck Surg 90: 108-116, 1982

13. Ojemann RG, Levine RA, Montgomery WM, McGaffigan PM: Use of intraoperative auditory evoked potentials to preserve hearing in unilateral acoustic neuromas removal. J Neurosurg 61: $938-948,1984$

14. Sugita K, Kobayashi S: Technical and instrumental improvements in the surgical treatment of acoustic neurinomas. J Neurosurg 57: 747-752, 1982

15. Møller AR, Jannetta PJ: Monitoring facial EMG responses during microvascular decompression operations for hemifacial spasm. J Neurosurg 66: 681-685, 1987

16. Møller AR, Møller MB: Does intraoperative monitoring of auditory evoked potentials reduce incidence of hearing loss as a complication of microvascular decompression of cranial nerves? Neurosurgery 24: 257-263, 1989

17. Prass R, Lueders H: Acoustic (loudspeaker) facial electromyographic monitoring. Part I. Neurosurgery 19: 392-400, 1986

18. Delgado TE, Buchheit WA, Rosenholtz HR, Chrissian S: Intraoperative monitoring of facial muscle evoked responses obtained by intracranial stimulation of the facial nerve: A more accurate technique for facial nerve dissection. J Neurosurg 4 : 418-421, 1979

19. Møller AR, Jannetta PJ: Hemifacial spasm: Results of electrophysiologic recording during microvascular decompression operations. Neurology 35: 969-974, 1985

20. Silverstein $\mathbf{H}$, Norrel $\mathrm{H}$, Hyman S: Simultaneous use of $\mathrm{CO} 2$ laser with continuous monitoring of eighth cranial nerve action potential during acoustic neuroma surgery. Otolaryngol Head Neck Surg 92: 80-84, 1984

21. Hardy RW Jr, Kinney SE, Lueders H, Lesser RP: Preservation of cochlear nerve function with aid of brain stem auditory evoked potentials. Neurosurgery 11: 16-19, 1982

22. Levine RA, Montgomery WW, Ojemann RG, Springer MFB: Evoked potential detection of hearing loss during acoustic neuroma surgery. Neurology 28: 339, 1978

23. Møller AR, Jannetta PJ: Monitoring auditory functions during cranial nerve microvascular decompression operations by direct recording from the eighth nerve. J Neurosurg 59: 493-499, 1983

24. Møller AR: Use of zero-phase digital filters to enhance brainstem auditory evoked potentials (BAEPs). Electroencephalogr Clin Neurophysiol 71: 226-232, 1988 